\title{
Eficácia da técnica de sutura ajustável per-operatória simplificada para a correção de desvios horizontais: estudo prospectivo de 49 pacientes
}

\author{
The efficacy of a simplified intraoperative adjustable suture for horizontal strabismus \\ surgery:a prospective study of 49 patients
}

\author{
Mariza Polati ${ }^{1}$ \\ Cíntia Fabiane Gomi² \\ Carlos Alberto Rodrigues Alves ${ }^{3}$ \\ SandradeFátima Medeiros Reis Verderosi ${ }^{4}$
}

${ }^{1}$ Médica Assistente, Chefe do Serviço de Motilidade Ocular Extrínseca e Pós-Graduanda do Departamento de Oftalmologia da Faculdade de Medicina da Universidade de São Paulo.

${ }^{2}$ Médica Estagiária do Serviço de Motilidade Ocular Extrínseca do Departamento de Oftalmologia da Faculdade de Medicina da Universidade de São Paulo.

${ }^{3}$ Professor Associado e Chefe do Serviço de NeuroOftamologia do Departamento de Oftalmologia e Otorrinolaringologia da Faculdade de Medicina da Universidade de São Paulo.

${ }^{4}$ Médica Assistente do Departamento de Anestesiologia do Hospital das Clínicas da Faculdade de Medicina da Universidade de São Paulo.

Endereço para correspondência: Mariza Polati - Rua Mello Alves, 640 - apto. 171 - São Paulo (SP)

CEP 01417010 - E-mail: mpolati@link.com.br

Recebido para publicação em 13.05.2002

Aceito para publicação em 30.10.2002

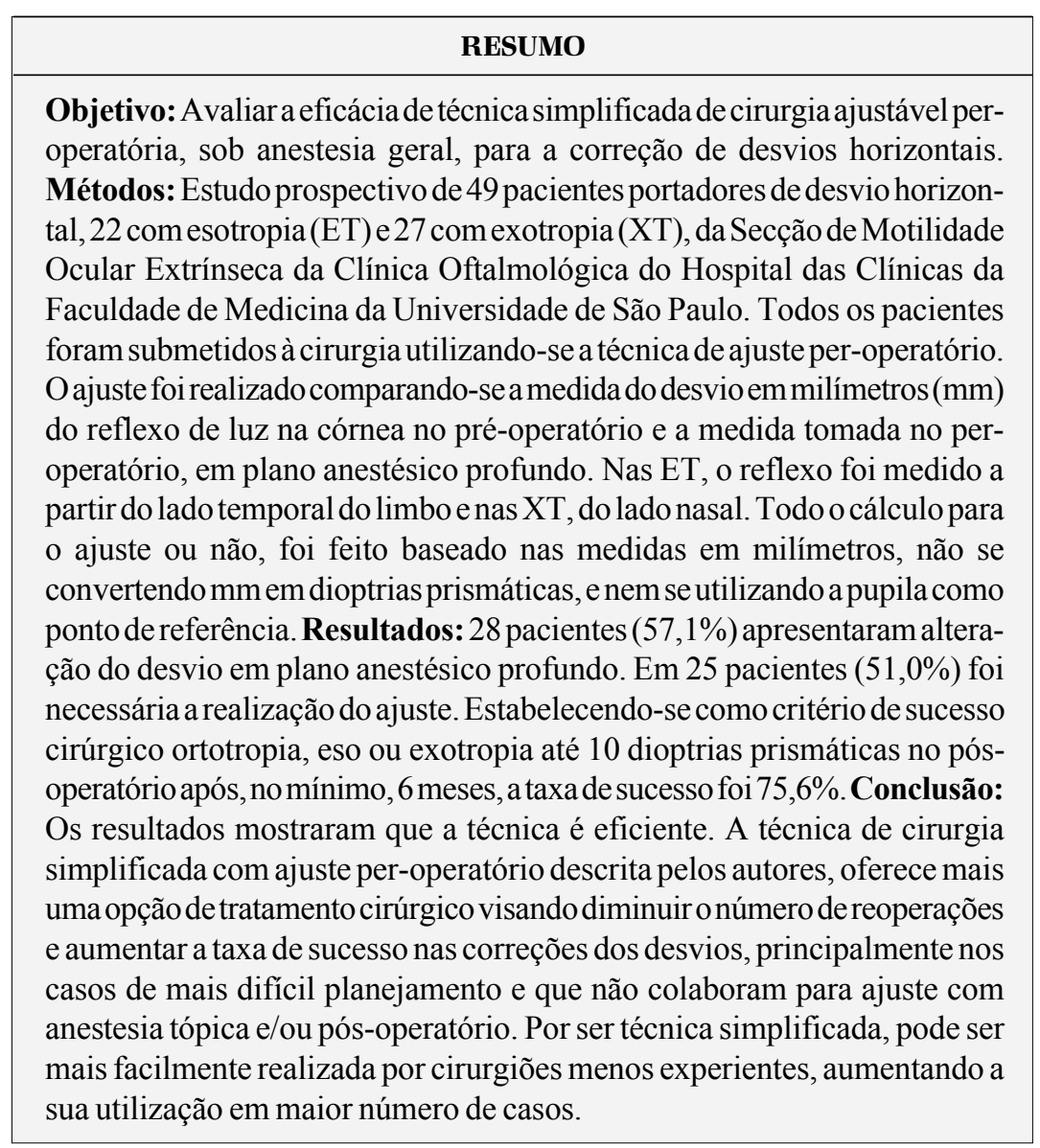

Descritores: Técnicas de sutura; Anestesia geral; Estrabismo/cirurgia; Esotropia/cirurgia; Exotropia/cirurgia; Estudos prospectivos; Adulto

\section{INTRODUÇÃO E OBJETIVO}

Muitos esforços têm sido feitos visando reduzir o número de reoperações e aumentar a taxa de sucesso das cirurgias de estrabismo. Sugere-se que o fato de realizar-se a cirurgia sobre tecidos moles, como os músculos extra-oculares, e a dificuldade em determinar referências anatômicas precisas, sejam fatores que contribuam para o insucesso em cerca de $20 \%$ dos $\operatorname{casos}^{(1)}$. 
A mesma cirurgia planejada para pacientes distintos com desvios semelhantes, muitas vezes, resulta em correções pósoperatórias diferentes, tornando-se difícil predizer o resultado final para o paciente individual. Esta situação é mais problemática nos portadores de alterações anatômicas ou decorrentes da moléstia a ser tratada, ou provindas de cirurgias prévias, de estrabismo ou orbitária.

Com o objetivo de melhorar a previsibilidade dos resultados, principalmente nesses casos de difícil tratamento, Jampolski, em 1979, popularizou a técnica de cirurgia com sutura ajustável ${ }^{(2)}$. Para a realização desta cirurgia, introduziu uma nova técnica de retrocesso muscular, chamada pelos autores americanos de "hang loose" ou "hang back", aqui traduzida por sutura em rédea.

Capó et al. e Repka et al. modificaram a técnica e demonstraram a utilidade e a maior segurança deste tipo de sutura ${ }^{(3-4)}$. Repka et al. compararam dois grupos de pacientes com esotropia, usando em um grupo a técnica de sutura escleral fixa e no outro a técnica de sutura em rédea ${ }^{(5)}$. Verificaram que o resultado cirúrgico foi muito semelhante. A técnica de sutura em rédea passou então a ser preferida nas cirurgias de retrocesso muscular porque permite:

- modificar a quantidade de cirurgia, seja no per ou no pósoperatório. Há a opção de ajuste.

- efetuar grandes retrocessos com menor risco de perfuração escleral, pelas próprias características da técnica.

Por outro lado, a cirurgia ajustável poderá compensar, ao menos de forma parcial, alguns fatores pouco previsíveis, mas que interferem nos resultados cirúrgicos. Entre eles citam-se: a anestesia geral altera a posição dos olhos ${ }^{(5,6-8)}$ e modifica a referência para os músculos. Dados eletromiográficos de retos mediais, sob anestesia geral, revelaram que a resposta muscular estava quase totalmente abolida no estágio em que os olhos estavam numa posição estável ${ }^{(9)}$. Drogas per-operatórias podem interferir no estado muscular, como a succinilcolina, por exemplo. Esta droga é relaxante muscular despolarizante, que facilita a entubação, mas provoca contração dos músculos extrínsecos, que pode ser imprevisível, e que persiste pelo menos por 15 minutos após a injeção $0^{(10-11)}$.

Atualmente, a cirurgia ajustável pode ser usada tanto para casos de estrabismo primário como naqueles em que participam fatores mecânicos passivos e fatores inervacionais ativos, nos quais o resultado cirúrgico é mais imprevisível com as técnicas tradicionais. A cirurgia ajustável é indicada em: reoperações, estrabismos paralíticos, casos com limitação mecânica severa (orbitopatia tireoidiana, síndromes de fibrose), inervação aberrante ou excessiva, anomalias inervacionais congênitas (síndromes de Duane e Ciancia), estrabismos de grande ângulo e estrabismo sensorial.

Realiza-se também o ajuste per-operatório nos casos em que o resultado é imprevisível, nos de difícil planejamento no pré-operatório e nos pacientes em que o ajuste pós-operatório é impraticável (pacientes pouco colaborativos e principalmente crianças).
Como o que se busca é o bom resultado cirúrgico, com estabilidade a longo prazo, alguns fatos já conhecidos devem ser observados, pois eles podem antecipar uma eventual modificação futura:

- A modificação do resultado cirúrgico ocorre com menos freqüência após cirurgias monoculares de retrocesso - ressecção ${ }^{(12)}$.

- Nos casos de esotropia e hipertropia há igual tendência de deslocamento dos olhos na mesma ou na direção contrária do desvio pré-operatório ${ }^{(12)}$.

- Nas exotropias é maior a tendência de deslocamento na direção do desvio original ${ }^{(12)}$.

Esta pesquisa apresenta técnica de sutura ajustável peroperatória simplificada para a correção dos desvios horizontais, com o objetivo de tentar diminuir o número de reoperações e aumentar a taxa de sucesso no resultado das cirurgias.

\section{MÉTODOS}

Foram selecionados para este estudo prospectivo os portadores de estrabismo horizontal com indicação cirúrgica, acompanhados no ambulatório de Motilidade Ocular Extrínseca da Clínica Oftalmológica do Hospital das Clínicas da Faculdade de Medicina da Universidade de São Paulo, no período de 1995 a 1999.

Excluíram-se os pacientes com esotropia acomodativa, os com diferença de desvio longe e perto maior que 10 dioptrias prismáticas (DP), anisotropias, hipertropias e desvio vertical dissociado.

Foi realizada a cirurgia ajustável em 49 pacientes, com idade entre 6 e 55 anos (média $=23$ anos e 3 meses \pm 12 anos e 3 meses, mediana $=20$ anos). Dos 49 pacientes operados, 22 $(44,9 \%)$ tinham esotropia (ET) e $27(55,1 \%)$ exotropia (XT). Considerando as subclassificações dos desvios, a amostra de 49 pacientes subdivide-se nos tipos de desvio descritos na tabela 1 .

Na tabela 2, encontra-se a distribuição das causas da baixa acuidade visual, quando presente, em um ou nos dois olhos.

Todos os pacientes foram submetidos a exame oftalmológico completo: medida da acuidade visual, exame ocular externo, refratometria sob cicloplegia (ciclopentolato), biomicroscopia, fundoscopia, medida da pressão intra-ocular e exame de motilidade ocular extrínseca (avaliação motora, sensorial quando necessária e aspecto estético).

\begin{tabular}{|c|c|c|c|}
\hline \multicolumn{2}{|c|}{ Tipo de desvio } & $\mathbf{n}$ & $\%$ \\
\hline \multirow[t]{3}{*}{ ET } & Infantil (até 6 m de idade) & 5 & 10,2 \\
\hline & Adquirida & 14 & 28,6 \\
\hline & Parética & 3 & 6,1 \\
\hline \multirow[t]{2}{*}{ XT } & Consecutiva & 8 & 16,3 \\
\hline & Básica & 19 & 38,8 \\
\hline Total & & 49 & 100,0 \\
\hline
\end{tabular}




\begin{tabular}{|lcr|}
\hline \multicolumn{2}{|c|}{$\begin{array}{c}\text { Tabela 2. Distribuição dos } \\
\text { causas de baixa pacientes operados segundo as }\end{array}$} \\
Causa & $\mathbf{n}$ & $\%$ \\
Ambliopia por estrabismo & 9 & 18,4 \\
Ambliopia por anisometropia & 5 & 10,2 \\
Catarata congênita unilateral & 2 & 4,1 \\
Catarata congênita bilateral & 3 & 6,1 \\
Coriorretinite macular & 3 & 6,1 \\
Alta miopia & 3 & 6,1 \\
Descolamento de retina & 1 & 2,0 \\
Leucoma de córnea & 1 & 2,0 \\
Alteração nervo óptico & 1 & 2,0 \\
Sem baixa acuidade visual & 21 & 42,9 \\
Total & 49 & 100,0 \\
\hline
\end{tabular}

Mediram-se os ângulos dos desvios na posição primária do olhar (PPO) e a $33 \mathrm{~cm}$, nas posições extremas do olhar, em supra e infraversão, sempre com o paciente portando correção óptica adequada. Os métodos utilizados para a avaliação préoperatória do desvio foram:

1) Teste de prisma e "cover" alternado: em pacientes com acuidade visual suficiente para permitir boa fixação ao alvo acomodativo.

2) Teste de Krimsky (original e/ou modificado): método usado em pacientes amblíopes, em pacientes sem fixação central ou em crianças que não colaboraram para o teste de prisma e "cover".

3) Avaliação das versões e ducções.

4) Medida do reflexo de luz sobre a córnea: medida em milímetros com régua, a partir do limbo. Convencionou-se medir o reflexo a partir do lado temporal do limbo nas esotropias (ET) e do lado nasal nas exotropias (XT). Para a medida deste reflexo, o observador se coloca em frente ao paciente, a uma distância de $2 / 3$ de metro. Coloca-se um foco de luz na direção da linha que une o dorso do nariz do médico ao dorso do nariz do examinado. Faz-se a medida no olho direito com o olho esquerdo do observador aberto e o direito fechado. De modo análogo, faz-se a medida no olho esquerdo e depois com os dois olhos do examinador abertos.

5) Medida do diâmetro corneano: foi realizada em todos os casos.

Os pacientes encaminhados para a cirurgia foram submetidos à avaliação clínica pré-operatória, exames laboratoriais (hemograma, coagulograma) e eletrocardiograma nos pacientes adultos. Outros exames cabíveis foram realizados para casos especiais.

Todas as cirurgias foram realizadas pelo mesmo cirurgião (M.P.) e todos os pacientes foram anestesiados pelo mesmo anestesiologista (S.F.M.R.V.).

\section{ANESTESIA GERAL PARA A CORREÇÃO DE ESTRABISMO}

Após a avaliação pré-anestésica, foi realizada a monitorização com o eletrocardiograma e verificada arritmia de pulso.

Em crianças com até $20 \mathrm{~kg}$, realizou-se a indução anestésica com máscara, utilizando óxido nitroso e oxigênio e introduzindo gradativamente uma droga halogenada (Sevorane). Potencializou-se a indução com Fentanil. Isto permitiu conseguirmos a entubação orotraqueal sem a necessidade de relaxantes musculares, na maioria dos casos. A manutenção da anestesia foi feita, sob respiração espontânea assistida manualmente, com óxido nitroso, oxigênio e Sevorane, podendo repetir o Fentanil se necessário.

Em crianças com peso de 20 a $40 \mathrm{~kg}$, a indução foi realizada com Fentanil e Propofol e ventilação manual com óxido nitroso, oxigênio e Sevorane. A manutenção foi feita com respiração mecânica controlada com óxido nitroso, oxigênio e Sevorane, mantendo-se ligeira hiperventilação, o que dispensa o uso de relaxantes musculares.

Quando a freqüência cardíaca permitiu, foi utilizada atropina para evitar sua alteração quando a musculatura ocular extrínseca era tracionada (reflexo óculo-cardíaco).

Em adultos, foi utilizada medicação pré-anestésica: Inoval, atropina (em pacientes sem contra-indicação) e Midazolan. A indução foi feita com Propofol ou Etomidato. A manutenção foi realizada sob respiração mecânica controlada com óxido nitroso, oxigênio e halogenado (Sevorane ou Forane).

Ao final da cirurgia, todos os pacientes foram medicados com dipirona para complementação da analgesia (exceto nos casos com história prévia de reação alérgica à droga).

\section{TÉCNICA DE AJUSTE PER-OPERATÓRIO SIMPLIFICADO}

Aguarda-se no mínimo 10 minutos após o início da anestesia para fazer-se a avaliação do desvio. Somente em plano anestésico profundo os olhos atingem a posição estável de repouso.

Os focos cirúrgicos são desligados, mas a iluminação da sala cirúrgica é mantida.

A medida do reflexo de luz na córnea é feita com o cirurgião posicionado na cabeceira da mesa cirúrgica, imediatamente atrás da cabeça do paciente. Usa-se um foco de luz (Penlight Welch Allyn 76 600) mantido a 1 metro do dorso do nariz do paciente. Os olhos do cirurgião ficam a $2 / 3$ de metro, aproximadamente, dos olhos do paciente.

O cirurgião afasta manualmente as pálpebras para a medida do reflexo. O reflexo de luz na córnea do olho fixador deve coincidir em milímetros com a medida realizada no pré-operatório, mesmo que para isso se tenha que mover a cabeça do paciente lateralmente. Mantém-se a cabeça nesta posição e mede-se então o reflexo no olho desviado. Avalia-se a seguir a diferença da medida entre o pré e o per-operatório. Esta diferença será importante no cálculo do ajuste. O ajuste sempre é realizado sobre o músculo retrocedido; raramente se ajusta o músculo ressecado.

Após as medidas, efetua-se a prova das ducções forçadas passivas para estudar eventuais limitações do movimento ocular no per-operatório.

Como calcular o ajuste:

1) Medir o reflexo luminoso na córnea dos dois olhos a partir do limbo no pré-operatório. 
2) Sob anestesia geral, repetir as medidas e estabelecer a diferença entre a medida no pré-operatório e em plano anestésico no olho desviado.

Duas situações podem ocorrer:

1) Não haver modificação do desvio em plano anestésico. Nesta situação, se o que se planeja é ortotropia, por exemplo, ao final da cirurgia a medida do reflexo no olho antes desviado deve estar na mesma distância do limbo que no olho fixador (Diagrama 1).

2) Haver modificação do desvio no per-operatório. Deve ser considerada a diferença da medida do reflexo no pré e no per-operatório do olho desviado. Ao final da cirurgia, se o que se planeja é ortotropia, o reflexo no olho antes desviado deve estar na mesma distância do limbo que no olho fixador, acrescida da diferença encontrada nos casos de esotropia (Diagrama 2) e subtraída nos casos de exotropia (Diagrama 3).

Em todos os casos, a cirurgia foi feita inicialmente conforme o planejamento pré-operatório, baseada na medida do desvio, e todos os cálculos de ajuste foram realizados após a realização deste procedimento. Deve-se sempre considerar para o ajuste o resultado final desejado, se ortotropia, super ou subcorreção, do desvio inicial.

Antes de realizar-se a medida do reflexo na córnea e o ajuste, após ter sido feito o que se planejou no pré-operatório, faz-se o teste de equilíbrio de forças, chamado por Jampolsky

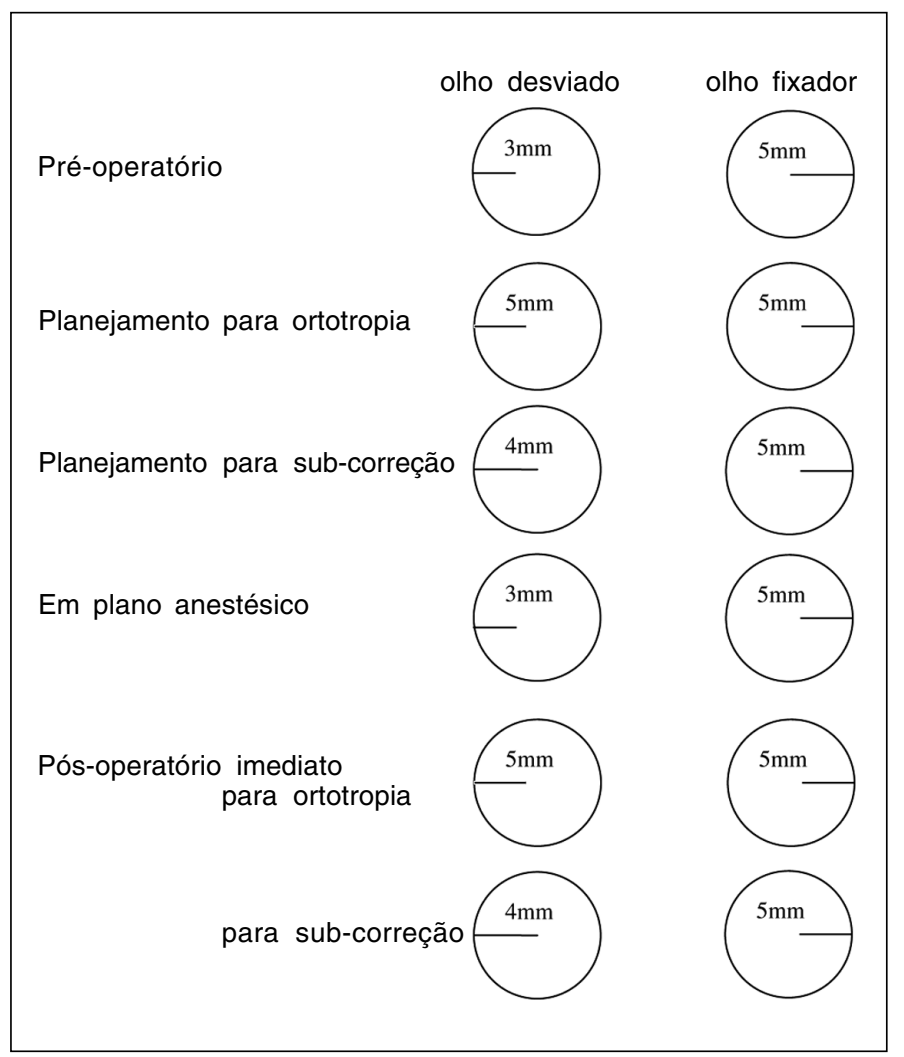

Diagrama 1 - Exemplo: Paciente com esotropia, sem modificação da medida do reflexo luminoso na córnea no pré e per-operatório como "spring back balance test"(13), que consiste em realizar rotações passivas repetidas para adução máxima e abdução máxima, e observar a posição de repouso que se segue a estas rotações. $\mathrm{O}$ teste tem como finalidade aliviar a tensão exercida

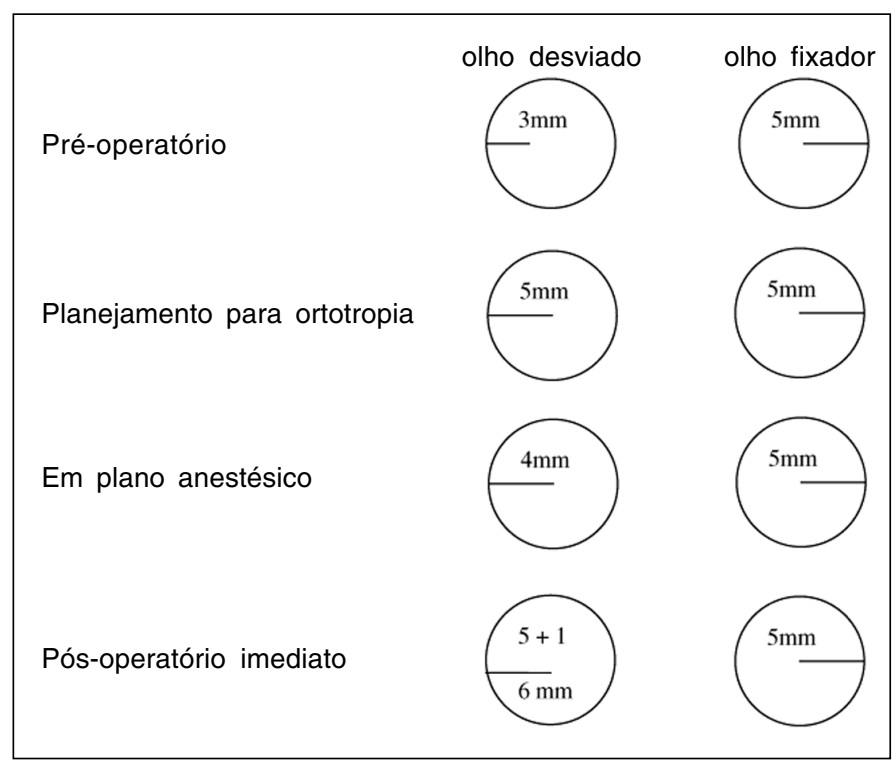

Diagrama 2 - Exemplo: Paciente com esotropia com relaxamento do desvio em plano anestésico profundo

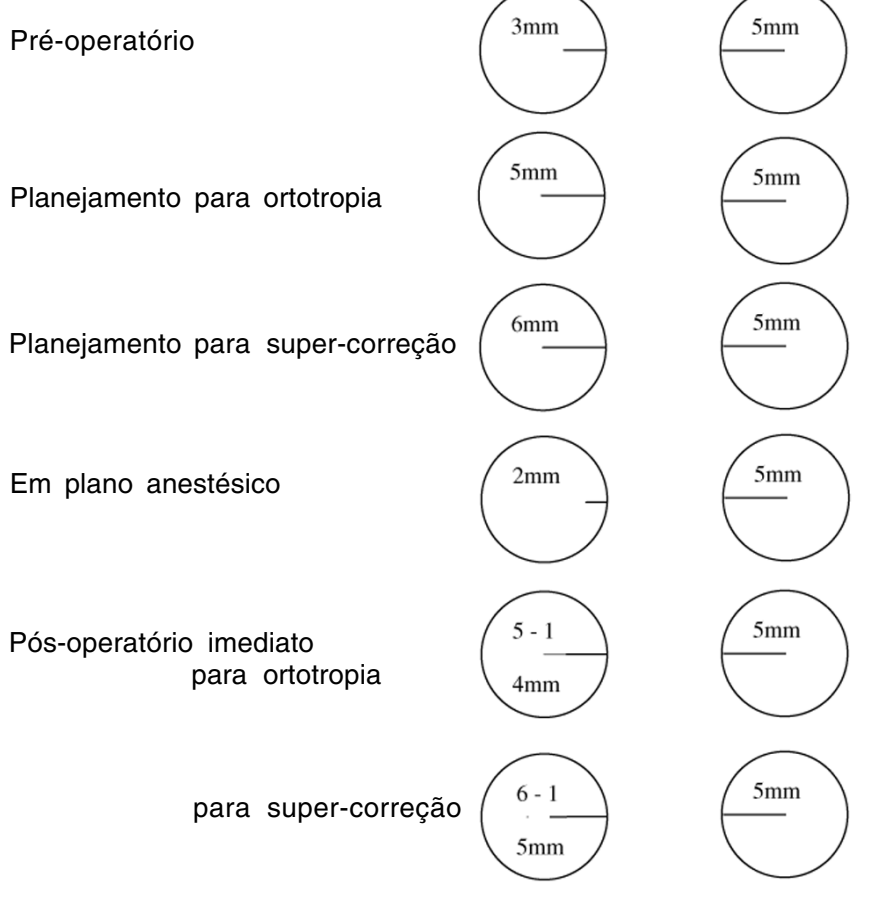

Diagrama 3 - Exemplo: Paciente com esotropia, com aumento do desvio em plano anestésico 
sobre os músculos operados pela tração necessária para proceder-se à cirurgia e relaxar as forças passivas, representadas pelas fascias, ligamentos e tecido perimuscular.

Considerou-se como bom resultado cirúrgico, ortotropia e eso ou exodesvio até $10 \mathrm{DP}$, após seguimento mínimo de 6 meses pós-operatórios.

\section{RESULTADOS}

Durante a cirurgia, $28(57,1 \%)$ dos 49 pacientes operados apresentaram alteração do desvio em plano anestésico. Desses 28 casos, 6 (21,4\%) tinham XT com aumento do desvio, 5 (17,9\%) apresentavam XT com diminuição do desvio e $17(60,7 \%)$ tinham ET com diminuição do desvio em plano anestésico.

Na tabela 3, encontra-se a distribuição das variações do desvio apresentadas pelos 28 pacientes em plano anestésico.

Encontrou-se associação estatisticamente significante entre tipo de desvio e sua alteração em plano anestésico $(\mathrm{P}=0,0226)$. Este fato foi de particular evidência entre os portadores de ET. A modificação do ângulo do estrabismo sob anestesia ocorreu em 77,3\% das ET e 40,7\% das XT. Houve diferença significante entre essas proporções (Tabela 4).

No per-operatório, em 25 casos $(51,0 \%)$ foi necessário o ajuste, que não foi realizado nos outros $24(49,0 \%)$.

Os resultados pós-operatórios foram analisados com um período médio de seguimento de 12 meses e 9 dias $(\mathrm{dp}=6$ meses e 24 dias), variando de 6 meses a 2 anos, com mediana de 12 meses.

Obtivemos os seguintes resultados pós-operatórios: 16 $(32,7 \%)$ casos de ortotropia, 21 (42,9\%) com sub ou supercorreção até 10 DP, $6(12,2 \%)$ com sub ou supercorreção entre 10 e 15 DP e $6(12,2 \%)$ casos com sub ou supercorreção acima de $15 \mathrm{DP}$.

\begin{tabular}{|c|c|c|}
\hline Variação do desvio & $\mathbf{n}$ & $\%$ \\
\hline $1,0 \mathrm{~mm}$ & 5 & 17,9 \\
\hline $2,0 \mathrm{~mm}$ & 15 & 53,6 \\
\hline $3,0 \mathrm{~mm}$ & 6 & 21,4 \\
\hline $4,0 \mathrm{~mm}$ & 1 & 3,6 \\
\hline $5,0 \mathrm{~mm}$ & 1 & 3,6 \\
\hline Total & 28 & 100,0 \\
\hline
\end{tabular}

\begin{tabular}{|c|c|c|c|c|}
\hline \multirow{2}{*}{$\begin{array}{l}\text { Alteração do desvio } \\
\text { em plano anestésico }\end{array}$} & \multicolumn{2}{|c|}{ Desvio ET } & \multicolumn{2}{|c|}{ Desvio XT } \\
\hline & $n$ & $\%$ & $\mathrm{n}$ & $\%$ \\
\hline Não & 5 & 22,7 & 16 & 59,3 \\
\hline Sim & 17 & 77,3 & 11 & 40,7 \\
\hline Total & 22 & 100,0 & 27 & 100,0 \\
\hline
\end{tabular}

Dos 16 pacientes com ortrotopia, $10(62,5 \%)$ apresentavam ET no pré-operatório e $6(37,5 \%)$ tinham XT. Nesse grupo, em 8 deles $(50,0 \%)$ foi necessário o ajuste per-operatório, dos quais 4 tinham ET e 4 XT.

Dos 21 pacientes com super ou subcorreção até $10 \mathrm{DP}, 5$ $(23,8 \%)$ apresentavam ET no pré-operatório e $16(76,2 \%)$ XT. $\mathrm{O}$ ajuste per-operatório foi feito em $10(47,6 \%)$ pacientes, dos quais 4 apresentavam ET e 6 XT.

No grupo de 6 pacientes com sub ou supercorreção de 10 a $15 \mathrm{DP}$, todos ficaram subcorrigidos no pós-operatório. $\mathrm{O}$ ajuste foi necessário em $4(66,7 \%)$ casos, dos quais 1 tinha ET e 3 XT. No pré-operatório, $2(33,3 \%)$ apresentavam ET e 4 (66,7\%) XT.

Nos 6 casos com sub ou supercorreção acima de 15 DP, $5(83,3 \%)$ tinham ET e $1(16,7 \%)$ XT no pré-operatório. Desses pacientes, em $3(50,0 \%)$ foi feito ajuste, dos quais 2 tinham ET e 1 XT.

Dos 49 pacientes operados, $9(18,4 \%)$ apresentaram visão binocular no pós-operatório ("Titmus de 3000 a 40" de arco). Desses 9 pacientes, $6(66,7 \%)$ tinham XT e $3(33,3 \%)$ ET.

$\mathrm{Na}$ tabela 5, encontra-se a distribuição dos valores dos desvios pré-operatórios (Krimsky ou prisma e "cover"). Dos 49 pacientes, $25(51,0 \%)$ apresentavam desvio igual ou maior que 45 DP e $24(49,0 \%)$ tinham desvio até 40 DP.

No grupo com desvio igual ou maior que 45 DP, $20(80,0 \%)$ obtiveram sucesso cirúrgico e $5(20,0 \%)$ foram considerados como insucesso. Dos pacientes com sucesso, $10(50,0 \%)$ tinham ET, dos quais em 7 foi feito ajuste no per-operatório, e 10 $(50,0 \%)$ tinham XT, sendo que em 6 deles foi necessário o ajuste. Todos os 5 casos considerados como insucesso apresentavam ET no pré-operatório e em 2 deles foi necessário o ajuste per-operatório.

No grupo com desvio até 40 DP, $23(95,8 \%)$ obtiveram sucesso cirúrgico e $1(4,2 \%)$ foi considerado como insucesso. Dos 23 casos com sucesso, 7 (30,4\%) tinham ET, em 2 foi necessário o ajuste per-operatório, e $16(69,6 \%)$ apresentavam $\mathrm{XT}$, sendo que o ajuste foi feito em 7 pacientes. O caso com insucesso tinha XT e foi feito o ajuste per-operatório.

Não foi encontrada associação estatisticamente significante entre alteração do desvio no per-operatório e necessidade de ajuste per-operatório $(\mathrm{P}=0,4832)$ (Tabela 6).

\begin{tabular}{|ccr|}
\hline \multicolumn{3}{|c|}{$\begin{array}{c}\text { Tabela 5. Distribuição dos } \\
\text { do desvio pacientes operados segundo o valor }\end{array}$} \\
Valor do desvio & $\mathbf{n}$ & $\%$ \\
$20^{\Delta}$ & 1 & 2,0 \\
$25^{\Delta}$ & 1 & 2,0 \\
$30^{\Delta}$ & 6 & 12,2 \\
$35^{\Delta}$ & 4 & 8,2 \\
$40^{\Delta}$ & 12 & 24,5 \\
$45^{\Delta}$ & 4 & 8,2 \\
$50^{\Delta}$ & 4 & 8,2 \\
$55^{\Delta}$ & 6 & 12,2 \\
$60^{\Delta}$ & 4 & 8,2 \\
$65^{\Delta}$ & 1 & 2,0 \\
$\geq 70^{\Delta}$ & 6 & 12,2 \\
Total & 49 & 100,0 \\
\hline
\end{tabular}




\begin{tabular}{|c|c|c|c|c|}
\hline \multirow[t]{2}{*}{$\begin{array}{c}\text { Ajuste } \\
\text { per-operatório }\end{array}$} & \multicolumn{2}{|c|}{$\begin{array}{c}\text { Alteração do } \\
\text { desvio ausente }\end{array}$} & \multicolumn{2}{|c|}{$\begin{array}{c}\text { Alteração do } \\
\text { desvio presente }\end{array}$} \\
\hline & $n$ & $\%$ & $\mathbf{n}$ & $\%$ \\
\hline Não & 12 & 57,1 & 12 & 42,9 \\
\hline Sim & 9 & 42,9 & 16 & 57,1 \\
\hline Total & 21 & 100,0 & 28 & 100,0 \\
\hline
\end{tabular}

\section{DISCUSSÃO}

Dos resultados obtidos, pode-se observar:

- Pacientes com ET tendem a alterar o desvio, em plano anestésico, com mais freqüência $(77,3 \%)$ que os com XT $(40,7 \%)$. Caldeira observou que a modificação do desvio durante a anestesia geral foi mais comum nos seus casos de XT $(45,7 \%)$ que nos casos de $\operatorname{ET}(10, \%)^{(6-7)}$.

- O ajuste per-operatório foi necessário em $51,0 \%$ dos pacientes, havendo portanto necessidade de modificar-se o planejamento pré-operatório. Enfatiza-se que, embora nos outros $49,0 \%$ não tenha sido realizado o ajuste, em todos foi medido o reflexo no pré e no per-operatório, como descrito em Métodos, e somente após a avaliação dessas medidas é que se descartou a necessidade de ajuste.

- Considerando como sucesso cirúrgico ortotropia, eso ou exodesvio de até 10 DP após 6 meses de pós-operatório, no mínimo, obtiveram-se $75,6 \%$ dos casos com sucesso, semelhante à taxa encontrada por Romano et al. $(71,4 \%)^{(8)}$. Caldei$\mathrm{ra}^{(6-7)}$ registrou sucesso em $65,7 \%$ dos casos de XT e $65,0 \%$ dos casos de ET. Ambos os autores usaram a técnica de Apt \& Isenberg ${ }^{(9)}$.

- Nos 6 casos de insucesso, com desvio pós-operatório $>15$ DP, 5 ficaram com desvio até 20 DP, com aspecto estético satisfatório, e 1, portador de XT, permaneceu com desvio inalterado, embora tenha sido realizado o ajuste. O paciente tinha história prévia de traumatismo crânio-encefálico.

As técnicas cirúrgicas que utilizam as suturas ajustáveis evoluíram de modificações das técnicas previamente utilizadas. O alvo dessas modificações tem sido alcançar melhores resultados quanto ao objetivo cirúrgico de alinhamento ocular na posição primária do olhar e o equilíbrio das rotações oculares, através de número mínimo de cirurgias.

Atualmente, a cirurgia ajustável com maior possibilidade de sucesso é a cirurgia realizada com anestesia tópica. Alguns autores $^{(15)}$ defendem esse procedimento por ser seguro, tão efetivo para o ajuste como o realizado sob anestesia geral, e porque diminui o tempo de recuperação pós-anestésico e os cuidados de enfermaria. É necessária, porém, muito boa cooperação do paciente, o que dificulta a sua utilização em crianças, pacientes ansiosos, deficientes mentais, entre outros.

Na cirurgia realizada sob anestesia geral e com ajuste pósoperatório, também se esbarra na dificuldade da apropriada colaboração do paciente. Além disso, o trauma cirúrgico cau- sa inflamação e edema tanto do músculo operado como das estruturas orbitais adjacentes, que podem afetar a posição do bulbo e o tônus, o estiramento e a contratilidade do músculo operado, interferindo no ajuste. A reação inflamatória piora após a cirurgia e atinge o pico mais acentuado entre 12 e 24 horas, muitas vezes o período selecionado para a realização do ajuste. Romano ${ }^{(14)}$ observou que esta técnica não oferece resultados melhores que os obtidos usando-se a técnica convencional.

Contudo, a precisão de ajuste com essas técnicas está muito diminuída em pacientes em que o teste de "cover" está prejudicado pela má fixação, que pode ser decorrente de ambliopia profunda, doenças retinianas ou doenças do nervo óptico, isto é, em pacientes portadores de estrabismo sensorial.

Foram propostas as técnicas de ajuste per-operatório em crianças, as quais não colaboram para ajuste pós-operatório ou sob anestesia tópica, e principalmente para os casos com estrabismo sensorial, em que o ideal é a correção total do desvio, realizando-se a cirurgia somente no olho com baixa acuidade visual.

As técnicas amplamente empregadas têm sido as baseadas na fórmula original proposta por Apt e Isenberg ${ }^{(9)} \mathrm{e}$ a modificada por Romano ${ }^{(8,14)}$. Nelas, a medida do desvio é feita pelo método de Hirschberg, usando a pupila como referência.

Outra técnica proposta por Bedrossian ${ }^{(16)}$ transforma a medida do desvio pré-operatório de dioptrias prismáticas (DP) para milímetros (considerando $1 \mathrm{~mm}=15 \mathrm{DP}$ ). Ao final da cirurgia, o reflexo luminoso na córnea deve ter-se deslocado a mesma quantidade em milímetros que a calculada com base no desvio pré-operatório. Nesta técnica, não importa a posição dos olhos sob anestesia geral. O cirurgião deve ter sempre em mente esta correspondência $15 \mathrm{DP}=1 \mathrm{~mm}$ para calcular o ajuste e assim poder modificar a cirurgia, se necessário.

$\mathrm{Na}$ técnica proposta pelos autores, utilizando-se a medida do reflexo luminoso na córnea a partir do limbo, não existe interferência de alterações pupilares fisiológicas, medicamentosas ou anatômicas. As medidas também não são afetadas pelos diâmetros corneanos (desde que iguais em ambos os olhos), nem pelo ângulo"kappa". O instrumental exigido é mínimo: foco luminoso e régua milimetrada esterilizada. Como as medidas são realizadas pelo reflexo luminoso na córnea em milímetros a partir do limbo (medida objetiva), seja nasal, seja temporal, o ajuste pode ser facilmente realizado por cirurgiões menos experientes nas técnicas de cirurgia ajustável.

\section{CONCLUSÃO}

Os resultados obtidos mostraram que a técnica descrita e executada pelos autores foi eficiente para corrigir desvios horizontais e proporcionar resultado cirúrgico melhor e mais previsível, considerando-se que em mais da metade dos casos foi necessário modificar-se no per-operatório o planejamento feito no pré-operatório. 
Como é uma técnica simplificada e que exige um material mínimo, pode ser utilizada por cirurgiões não acostumados com as técnicas de cirurgia ajustável, ampliando o seu uso para maior número de casos, nos quais a cirurgia ajustável pode fornecer resultado mais previsível, evitando assim número maior de reoperações.

\section{ABSTRACT}

Purpose: To evaluate the efficacy of a simplified intraoperative adjustable suture technique, under general anesthesia (GA) proposed by the authors. Methods: A prospective study of 49 patients with horizontal strabismus, 22 esotropes and 27 exotropes. The same surgeon (MP) operated on all of the patients. The corneal light reflection was measured in millimeters $(\mathrm{mm})$ from the limbus preoperatively and under GA: the esotropes from the temporal border and the exotropes from the nasal border. The adjustment was made or not based on these two measures if there was or not a difference between them. The authors calculated the adjustment on the measures in $\mathrm{mm}$, not converting them in to prism diopters (PD) or taking the pupil as a reference point. Results: 28 patients $(57.1 \%)$ had the eye position modified under GA. In 25 of all of them $(51.0 \%)$ surgery was adjusted. Surgical success was defined as orthotropia, eso- or exodeviation up to $10 \mathrm{PD}$, in a follow-up period of at least 6 months. The overall success rate was 75.6\%. Conclusion: Results suggested this simplified procedure is effective. As an adjustable technique can conduct to more predictable outcomes, and being a simplified technique it can be employed in more cases, specially because it can be carried out by surgeons not used to the adjustable suture techniques.
Keywords: Suture techniques; Anesthesia general; Strabismus/surgery; Esotropia/surgery; Exotropia/surgery; Prospective studies; Adult

\section{REFERÊNCIAS}

1. Metz HS. Adjustable suture strabismus surgery. Ann Ophthalmol 1979;11: 1593-7.

2. Jampolsky A. Current techniques of adjustable strabismus surgery. Am J Ophthalmol 1979;88:406-18.

3. Capó H, Repka MX, Guyton DL. Hang back lateral rectus recessions for exotropia. J Pediatr Ophthalmol Strabismus 1989;26:31-4.

4. Repka MX, Fishman PJ, Guyton DL. The site of reattachment of the extraocular muscle following hang back recession. J Pediatr Ophthalmol Strabismus 1990;27:286-90.

5. Repka MX, Guyton DL. Comparison of hang back medial rectus recession with conventional recession. Ophthalmology 1988;95:782-7.

6. Caldeira JAF. Stage I intraoperative adjustment of eye muscle surgery for exodeviations based on eye position during general anesthesia: a prospective study of 35 patients. Binoc Vision \& Strabismus 1991;28(4):206-11

7. Caldeira JAF. Intraoperative adjustment of eye muscle surgery for esotropia based on eye position during general anesthesia: a prospective study of 40 patients. Binoc Vision \& Strabismus 1994;9(1):23-30

8. Romano P, Gabriel L. Intraoperative adjustment of eye muscle surgery. Correction based on eye position during general anesthesia. Arch Ophthalmol 1985;103:351-3.

9. Apt L, Isenberg S. Eye position of strabismus patients under general anesthesia. Am J Ophthalmol 1977;84:574-9.

10. Lingua R, Li KF, Tingley D, Fever W. Succinylcholine stimulated muscle tensions in human strabismus. Binoc Vision \& Strabismus 1992;7:15-24.

11. Breinin GM. The position of rest during anesthesia and sleep: electromyographic observations. Arch Ophthalmol 1957;57:323-6.

12. Wright KW. Practical aspects of the adjustable suture technique for strabismus surgery. Int Ophthalmol Clin 1989;29:10-5.

13. Jampolsky A. Spring back balance test. In Strabismus. Symposium of the New Orleans Academy of Ophthamology. St Louis, V. Mosby Co, 1978, chap 7,147.

14. Romano P. Improved results in eye muscle surgery by intraoperative adjustment: the three methods (stages) applicable under general anesthesia. Semin Ophthalmol 1988;3:185-96.

15. Carruthers JD, Mills K, Bagaric D. Can adjustable suture surgery be performed with conscious sedation? J Pediatr Ophthalmol Strabismus 1995;32:17-9.

16. Bedrossian RH. Ädjusting adjustable eye muscle sutures in anesthetized patients. Ann Ophthalmol 1983;15:800,802-4.

\title{
ABO ELETRÔNICO
}

\author{
Novo site
}

\section{Acesso: http://www.abonet.com.br}

